\title{
Frühromantisches im Mittelaltergewand
}

Bis hierhin haben wir Strau $\beta$ folgen können, vielleicht allzu sehr bezaubert von seiner Sprache, seiner Belesenheit und dem schlechthin Zauberhaften des poetischen Melancholikers. Nun aber erfolgt eine Volte hinein ins Frühromantisch-Mittelalterliche, die möglicherweise denn doch verkennt, dass inzwischen der Elektromagnetismus den legitimen und "gerechtfertigten“ Nachfolger des Mesmerismus abgibt? Es erfolgt in Strauss' noch „electrischer“ Novellenwelt jedenfalls eine Identifizierung des „Deutschen“ mit dem Hochromantischen nach eher Heidelberger, als noch Jenaer Art. Mit Verve und Pathos wird eine unvergleichliche, weil weitgehend romantisch bestimmte „Deutsche Literatur“ gefeiert, die in Schriftstellern wie „Kleist, Novalis, Hofmannsthal“1 kulminiert hätte. Danach erfolgt der Salto (mortale? Wir werden sehen) hinein in's literarische Reservoir einer urdeutschen Hochromantik. sozusagen im Mondscheinschatten der Heidelberger Schlossruine durchgeführt. Titurel von Wolfram von Eschenbach und dessen Parzival geraten zu Ankerpunkten der Strauss'schen Erzählung. Sie werden es bleiben - bis in die novellistische Schluss-Volte hinein, in der die Grosse Mutter sich plötzlich und erschreckend zurückmeldet, sich spektakulär-archaisch resexualisierend. Und das, obwohl die Welt des Wolfram von Eschenbach bereits eine der durchaus respektierten Frauenehre, des ritterlichen Kampfes und vor allem - der „Minne“ gewesen ist; der „hohen“, wie auch der etwas weniger hohen. So ganz vermag der Paare, Passanten-Autor vom Eros nicht zu lassen, wie zu erwarten.

\footnotetext{
${ }^{1}$ Botho Strau $\beta$, Die Unbeholfenen. Bewusstseinsnovelle, a. a. O., S. 88.

(C) Der/die Autor(en) 2021

B. Neumann, Umrisse einer Dritten Kultur im interdisziplinären Zusammenspiel zwischen Literatur und Naturwissenschaft, ELECTRISCHER PROMETHEUS. Umrisse einer Dritten Kultur im interdisziplinären Zusammenspiel zwischen Literatur und Naturwissenschaft, https://doi.org/10.1007/978-3-662-63204-8_10
} 
Zuerst zum Titurel. Das Epos war Wolframs letztes Werk, nur lückenhaft erhalten, vermutlich um 1220 entstanden und für den Thüringer Hof bestimmt. Sein Titel ist irreführend; das Mittelalter benannte manchmal literarische Werke nach der ersten in ihnen vorkommenden Person. Das war hier ein „Titurel“. Der freilich gar nicht handlungsbestimmend erscheint. Vielmehr: „Der Titurel erzählt die Geschichte von Sigune und Schionatulander, deren Ende aus dem Parzival bekannt war: Schionatulander findet im Frauendienst den Tod, er wird im ritterlichen Zweikampf getötet. Daraufhin weiht Sigune ihr ganzes Leben der Klage und der Trauer um den toten Freund; sie lässt sich in eine Klause einmauern und stirbt schliesslich dem Geliebten nach.“2 (Es geht hier um „Parzival“; erst Richard Wagners Oper wird dann die Schreibweise „Parsival“ durchsetzen). Was das zitierte Lexikon selbstverständlich ebenfalls weiss, hier freilich nicht erwähnt, während es bei dem Erotiker Strau $\beta$ von zentraler Bedeutung ist: Sigune ist auf dem Weg, sich dem Geliebten auch körperlich hinzugeben. Der stirbt aber, als er ihre letzte Bitte vor der Hingabe erfüllen will. Sein bedeutendster Frauendienst wird zu seinem letzten. Schionatulanders Tod entspringt aus folgender paradoxer Situation: Die Liebenden haben ihr Zeltlager auf einer Waldlichtung aufgeschlagen. „Schionatulander fängt einen entlaufenen Jagdhund ein, auf dessen Halsband und Führleine eine Inschrift steht (in kostbarer Diamantenschrift gehalten, B.N.) ... Bevor Sigune die Inschrift zu Ende gelesen hat, reisst der Hund sich los und entläuft in den Wald. In feierlicher Form verheisst Sigune dem Freund die volle Hingabe, wenn er ihr das Halsband zurückbringt. " 3 Bei diesem Vorhaben, einem nur scheinbar ungefährlichen „Minnedienst“, kommt der Mann um. Im Parzival steht geschrieben: „Ein Brackenseil brachte ihm den Tod.“4 Die Bracke wiederum hiess: „Gardevias“, also: „Achte auf den Weg“. Das Werk ist zudem Fragment, also „geheimnisvoll“ und Stoff für die romantische Dichtungstheorie - ebenso wie Kafkas durchweg fragmentarische Romane. Damit nicht genug. Trotz (oder wegen?) seines Fragment-Charakters entfaltete der Titurel eine ganz singuläre Wirkungsgeschichte, indem er eine Nachdichtung von immerhin 6000 Strophen erfuhr durch einen Sänger, von dem nur der Vorname auf die Nachwelt kam: Albrecht. Diesem relativ Unbekannten war dann mit seinem Parzival einer der grössten literarischen Erfolge des Spätmittelalters überhaupt beschieden. Und es sollte kein anderer als der bedeutendste Theoretiker

\footnotetext{
${ }^{2}$ Kindlers Lexikon der Weltliteratur, a. a. O., S. 811.

${ }^{3}$ Kindlers Lexikon der Weltiteratur, a. a. O., S. 812.

${ }^{4}$ Wolfram von Eschenbach, Parzival, 141, 16.
} 
der Frühromantik August Wilhelm Schlegel sein, der erkannte, dass Wolfram seinerseits lediglich die beiden alten Fragmente gedichtet hatte, die ihrerseits gar keine Antwort geben auf die Frage, ob die Liebenden denn hinreichend genau auf ihren Weg geachtet hätten. Der nachgedichtete Parzival dagegen ist uns vollständig in seinen 25810 Versen erhalten. Das Epos speist sich aus dem Stoffkreis der keltischen Sagen um König Arthus samt Gralssuche, Tafelrunde und Christi Seitenwunde, weshalb das Stück gar nicht daran vorbeikommen konnte, von Richard Wagner vertont zu werden. Wolframs Epos wiederum war seinerseits durch die erzählertheoretische Besonderheit gekennzeichnet, dass sein Autor sich stets zum Dialog stellt, sich mit dem Leser unterhält und mithin nicht in der gottgleichen Autorität des späteren ,auctorialen“ Erzählers verbleibt. Darin gleicht er, übrigens, dem Ich-Erzähler bei Strau $\beta$. Unter bestimmten Gesichtspunkten hat also bereits Wolfram ein „Bewusstseinsepos“ geschaffen: „Der Erzähler ... schafft ... eine zweite Erzählebene, auf der er sich mit seinen Zuhörern unterhält und Fragen an sie stellt oder an ihr Urteil appelliert."5 Statt auctorialen, distanzierten Erzählens ein elektromagnetisches Hin und Her der beteiligten Elementarteilchen im Rezeptions-Feld zwischen den Polen Dichtung einerseits und Hörer/Leser andererseits. „Dadurch werden die Zuhörer in die Dichtung hineingezogen und $\mathrm{zu}$ Mitspielern gemacht.... Diese unruhig springende, von Gegensätzen lebende und auf Überraschungen zielende Erzählweise hat Wolfram selbst als eine Eigentümlichkeit seines ,krummen" Stils bezeichnet", was dem erotomanen Erzähler Strau $\beta$ dann bis in die Tatsache hinein entgegenkam, dass auf diese Weise ,häufig die Tabuzone des Sexuellen“ berührt wurde. ${ }^{6}$ Im Mittelalter war Wolframs Epos die mit Abstand bekannteste und bedeutendste Dichtung. Sie hat denn auch eine ungewöhnlich breite und tiefe Rezeptionsspur nach sich gezogen, die mit ihrer Drucklegung von 1477 als dem überhaupt einzigen höfischen Epos (neben dem erwähnten Albrecht'schen Jüngeren Titurel, der aber ebenfalls als Werk Wolframs galt) dazu führte, dass der Dichter selbst zu einer Figur in Singspiel, Dichtung, zahllosen Romanen geriet. Die Nürnberger Meistersinger haben Wolfram als einen der Zwölf Alten Meister verehrt. Im 18. Jahrhundert verfasste Johann Jakob Bodmer die erste Parzival-Übersetzung. Karl Lachmanns kritische Textausgabe von 1833 wurde dann an Bekanntheit durch die berühmteste moderne Bearbeitung des Stoffs: Richard Wagners gleichnamige

\footnotetext{
${ }^{5}$ Kindlers Lexikon der Weltliteratur, a. a. O., S. 810.

${ }^{6}$ Kindlers Lexikon der Weltliteratur, a. a. O., S. 810.
} 
Oper, abgelöst und übertroffen. „Parzival“ wurde zu „Parsival“, nicht unbedingt zu seines Helden Bestem.

Es gibt also gute Gründe dafür, dass Strau $\beta$ ausgerechnet die mittelalterliche Erzählung vom Brakkenseil sich auswählte für seine Unbeholfenen. Hier hat Nadja, die mehrfach Begehrte, ihren Auftritt, die mit der Wahl ihres neuesten Liebhabers einen allzu Beholfenen sich ins verwunschene Haus geholt hat; einen, der am Ende sich dann nur noch für ihre unablässig twitternde, darin so ganz „moderne“ Schwester interessiert. Der Mann gibt bei Strau $\beta$ einen ironisch trivialen Ich-Erzähler, der, alles andere als ein „Bündler“, am Ende vom grausig wiedererstandenen Muttermythos eingeholt wird. Hat also Nadja den rechten Weg gewählt, das steht als Frage hinter der Erzählung von der Bracke „Gardevias“, wie sie ihrerseits die Bewusstseinsnovelle insgesamt abschliesst. Botho Strau $\beta$ erklärt jetzt „Die Deutschen“ zum romantischen Volk per excellence; und nennt als deren Dichter doch immer nur die der Wiener Moderne. Seine Figur Albrecht als eine Variante des Eschenbach-Nachdichters dagegen führt aus: „Was es im Deutschen an höherem Bewusstsein gab, war immer romantisch, gleichgültig zu welcher Epoche. Romantisch nenne ich alles, was lebt, um sich zu sehnen. Oder, wie in deiner Geschichte: alles, was unvollendet bleibt, halb gelesen, halb entschlüsselt, halb erkannt. Und einen unstillbaren Antrieb zurücklässt. ... Man möchte es auf die Spitze trieben und sagen: die Technik tröstet anscheinend den abendländischen Menschen für das schwere Schicksal eines untröstlichen Denkens. Sie überträgt eine Art kindlicher Funktionslust auf seinen Verstand.“7 Demnach wäre der stehend marschierende Fragmentroman-Schreiber Franz Kafka aus der magischen Stadt Prag mit ihrer Sehnsucht nach der Weltformel ein (Früh)Romantiker und selbst Märtyrer des ,abendländisch untröstlichen Denkens"?

Bei Strau $\beta$ jedenfalls liest man: „Ich werde bis zuletzt dem Wissen und dem Wissenwollen das Wort reden. Denn in die Wissenschaften allein hat sich das Ingenium der Menschheit eingenistet ... Andererseits bin ich davon überzeugt, dass dem Aufstieg des wissenschaftlichen Denkens eine natürliche oder übernatürliche Grenze gesetzt ist. Auch wenn es noch so märchenhaft klingt: ich stelle mir vor, dass das forschende und bis ins letzte sich selbst erforschende Denken eines Tages ... auf eine Formel stossen wird, die es in tiefe Trance versetzt. Eine magische Formel, nach der jenes Denken zuvor niemals suchte, die ihm vielmehr aus den eisigen Höhen der Theorien und Abstraktionen, aus sich

\footnotetext{
${ }^{7}$ Botho Strau $\beta$, Die Unbeholfenen. Bewusstseinsnovelle, a. a. O., S. 95.
} 
selbst heraus, entgegentritt und es wie durch Zauberwirkung ausser Kraft setzt.“ Solche Sätze qualifizieren durchaus für die Verleihung einer Ehrenbürgerschaft im „magischen“ Prag! Dazu fügt sich, dass nun, auch um die Welt der „Bündler“ als die der wahrhaft interdisziplinär Denkenden hervorzuheben, der, der dies vorträgt, und es ist nicht der Ich-Erzähler, sondern sein Rivale Romero, sich geradezu hineinstürzt in die besonders ,magische“ Sphäre von Astrologie, Astronomie und vor allem der neuesten, der Einstein-Heisenberg'schen Physik: „Die Menschen - oder eine empfängliche Vorhut von ihnen - werden dann von ihren Höchstgeschwindigkeiten augenblicklich in eine ungeheure Dehnung der Zeit hinüberwechseln ... Nur jeweils ein Brocken Sichtbares erscheint ihnen und schwebt langsam durch ihren Dämmer wie ein abgebremster Meteor."8 Man kann hier nur hoffen, dass Strauß' Meteor, anders als noch der Dürrenmatt'sche, seinen Einschlag moderat vollzieht. Denn derart soll sie nun Wirklichkeit werden, die Utopie von der naturwissenschaftlichen Begründung des Mystischen: „Aus dieser Nacht aber oder langsamen Zeit baut sich eine neue Periode des bildhaften Denkens auf ... Man könnte auch sagen: irgendwann wird die letzte, äusserste Entfaltung des Geistes erreicht, die kritische Grenze berührt, so dass er sich wieder einzufalten beginnt, sich zurückzieht in seine Implikate: Bilder, Symbole, Hieroglyphen, Pictogramme ..."9 Damit wäre ist die äusserste Grenze, die eben auch immer eine der blossen Spekulation zu sein droht, erreicht. Der novellistisch vorausgesagte grosse Umschwung tritt ein - ironisch genug als ,powercut“, der einen weiteren e-mail-Flirt des Nadja'schen Liebhabers mit deren Schwester Ilona, der „schönen Rohen“, unterbricht. Doch der in dieser Szene als profanlächerlicher „elektrischer Prometheus“ agiert, wird nunmehr „electrisch“ in den neu erstandenen (feministischen?) Mythos hineingescheucht. Am Ende bleibt offen, ob er diese Begegnung überstehen wird. „, Geh mit mir zu meiner Mutter!' lautete die nächste Nachricht, die mir die flinkesten Fingerspitzen der Welt schickten ..., Wenn wir zurückkommen, setzen wir da wieder an, wo sie unterbrochen haben“."10 „Wenn wir zurückkommen..." - dem novellistischen Umschlagscharakter dieser mustergültig-archetypischen „Bewusstseinsnovelle“ wird eben nur der gerecht, der solches „Wenn“ nicht zeitlich, sondern konditional liest. Aus dem Reich der, nunmehr im neuesten, weltumspannenden „web“ eingefangenen, dennoch unvermindert mythischen Urmutter gibt es bei Strau $\beta$

\footnotetext{
${ }^{8}$ Botho Strau $\beta$, Die Unbeholfenen. Bewusstseinsnovelle, a. a. O., S. 99.

${ }^{9}$ Botho Strau $\beta$, Die Unbeholfenen. Bewusstseinsnovelle, a. a. O., S. 100.

${ }^{10}$ Botho Strau $\beta$, Die Unbeholfenen. Bewusstseinsnovelle, a. a. O., S. 101.
} 
kein Entkommen mehr. So erfüllt sein Text die in ihm ganz zentral verhandelte Prophezeiungen der „Bündler“, die, entkleidet man sie vom Beiklang des rückwärtsgewandt „Bündlerischen“, darauf hinausläuft, dass nur noch die Synthese modernsten Wissens in einer „Dritten Kultur“ dem heutigen Bewusstsein zu neuer, höherer Einsicht zu verhelfen vermöchte. Notabene: Die Voraussetzung dabei ist immer die Ablösung des „Auctorialen Erzählens“, dieser Errungenschaft des europäischen 18. und 19. Jahrhunderts, durch eine „elektromagnetische“ Freisetzung der einzelnen Akteure, die dann eben nicht mehr auf feste Bahnen angewiesen wären in einem Zeitalter, das in der Auflösung dieser Bahnen, gesellschaftlich-historisch wie physikalisch-erkenntnistheoretisch, zu sich selbst gefunden hat. Aus dieser Perspektive gilt: Innerhalb der deutschen Literatur der Gegenwart ist es der Uckermärker Strau $\beta$, der die Nachfolge des Pragers Kafka am konsequentesten und literarisch begabtesten angetreten hat - indem er dessen Erzählen in neues, das Wesentliche hell beleuchtendes Licht gestellt hat in seiner Bewusstseinsnovelle als einem Seitenstück zum Kafkaesken Roman, wie er vor allem im Schloss vorliegt.

Open Access Dieses Kapitel wird unter der Creative Commons Namensnennung 4.0 International Lizenz (http://creativecommons.org/licenses/by/4.0/deed.de) veröffentlicht, welche die Nutzung, Vervielfältigung, Bearbeitung, Verbreitung und Wiedergabe in jeglichem Medium und Format erlaubt, sofern Sie den/die ursprünglichen Autor(en) und die Quelle ordnungsgemäß nennen, einen Link zur Creative Commons Lizenz beifügen und angeben, ob Änderungen vorgenommen wurden.

Die in diesem Kapitel enthaltenen Bilder und sonstiges Drittmaterial unterliegen ebenfalls der genannten Creative Commons Lizenz, sofern sich aus der Abbildungslegende nichts anderes ergibt. Sofern das betreffende Material nicht unter der genannten Creative Commons Lizenz steht und die betreffende Handlung nicht nach gesetzlichen Vorschriften erlaubt ist, ist für die oben aufgeführten Weiterverwendungen des Materials die Einwilligung des jeweiligen Rechteinhabers einzuholen.

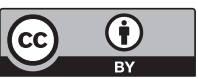

